\title{
Research report \\ Differential task effects on semantic and syntactic processes as revealed by ERPs
}

\author{
Anja Hahne*, Angela D. Friederici \\ Max Planck Institute of Cognitive Neuroscience, P.O. Box 500 355, D-04303 Leipzig, Germany
}

Accepted 20 November 2001

\begin{abstract}
Two experiments investigated the time-course of semantic and syntactic processes in auditory language comprehension as well as their possible functional dependencies, using event-related brain potentials (ERPs). Participants listened to sentences which were either correct, semantically incorrect, syntactically incorrect, or both semantically and syntactically incorrect. In experiment 1, participants judged the overall correctness of these sentences. The semantic violation elicited an N400 whereas the syntactic phrase structure violation elicited an early anterior negativity followed by a P600. Sentences in which the critical element violated both semantic and syntactic constraints elicited the same pattern of ERPs as the syntactic violation alone, not evoking an N400. In experiment 2, participants judged the same sentences for semantic coherence, required to ignore syntactic violations. Again, an early anterior negativity was elicited for those sentences containing phrase-structure errors. In contrast to experiment 1 , however, combined violations elicited both an early negativity and an N400. Together, the results suggest that the N400 associated with semantic aspects of sentence comprehension reflects controlled processes whereas initial parsing processes associated with the early anterior negativity are independent of semantic constraints and task requirements. (C) 2002 Elsevier Science B.V. All rights reserved.
\end{abstract}

Theme: Neural basis of behavior

Topic: Cognition: language

Keywords: Auditory event-related brain potential; Syntax; Semantics; N400

\section{Introduction}

In order for sentences to be understood, the human language comprehension system must process and integrate a wide range of heterogeneous linguistic information within a short time-period. The question of how the exact architecture of this system is best described, however, has yet to be definitively answered, in spite of the many models proposed to this extent during the last decades. While there appears to be a general consensus that sentence processing is incremental and that different types of information are processed fairly quickly whenever a new word is encountered [3,29], the proposed architectures differ with respect to when different information sources become available and/or are used, how they are coordi-

\footnotetext{
*Corresponding author. Tel.: +49-341-9940-230; fax: +49-341-9940113.

E-mail address: hahne@cns.mpg.de (A. Hahne).
}

nated and what their functional dependencies are $[9,11,28,29]$.

The present paper focuses on the processing of phrase structure (i.e. syntactic) and selectional restriction (i.e. semantic) information. In particular, we explore the temporal coordination of these two processes and, moreover, whether the processing of one type of information is a necessary prerequisite for the other to occur. In other words, we investigate whether the semantic integration of a word into a sentence depends on its prior successful integration at the phrase structure level and if so, whether such a primacy of structural aspects can be overcome by a task effecting an attentional shift to semantic aspects.

We examine these issues by using event-related brain potentials (ERPs) as the dependent variable. As ERPs are differentially sensitive to the various types of information in question and, furthermore, provide a continuous measure of sentence processing, this method seems particularly suited to an exploration of the issues at hand. The vast 
majority of ERP studies examining language comprehension has made use of the violation paradigm, in which responses to correct sentences are compared to those elicited by a particular syntactic and/or semantic violation. The observed difference is usually interpreted as reflecting the processing of the particular aspect varying between the two sentence types.

Before turning to our experimental design, we will briefly discuss some previous ERP studies on language processing with the focus being on those aspects of sentence comprehension which are relevant to the present study, namely selectional restriction and phrase structure processing. Semantic processes in reading were first examined in the seminal study by Kutas and Hillyard [26]. Here, it was demonstrated that semantically incongruent words in a sentence elicit a more negative going potential than their contextually congruent counterparts. This potential reached a maximum over centro-parietal scalp sites approximately $400 \mathrm{~ms}$ after the onset of the critical word and has thus been labeled N400 [27]. The N400 is not restricted to violations. Van Petten and Kutas [45] showed that, in correct sentences, a word's linear position is inversely correlated with the amplitude of the N400. This effect interacted with word frequency, i.e. frequency effects were observed only for positions early in the sentence. These data suggest that the N400 found in sentence processing is related to the semantic integration of a word into its sentential context.

During the last decade, ERP studies on language comprehension have also explored syntactic aspects of language processing. These studies have revealed two main types of ERP components, each associated with different types of syntactic processes, and observable either separately or conjointly. The first type is a negative potential varying in latency, but usually observable in a time domain below $500 \mathrm{~ms}$, while the second type is a positive potential with a peak latency that is usually greater than $500 \mathrm{~ms}$.

A particularly early negativity (appearing about $160 \mathrm{~ms}$ after the word onset) has been observed in correlation with phrase structure and word category violations [18,21$23,34]$. This negativity often had a maximum over the left anterior scalp and has therefore sometimes been labeled ELAN (early left anterior negativity). Gunter et al. [18] showed that, for visual presentation, the latency of the negativity depends on the quality of the visual input. While the negativity was present early $(150 \mathrm{~ms})$ using a high visual contrast, it was present only after about $450 \mathrm{~ms}$ when the stimuli were of low visual contrast. For spoken sentences, the latency of the effect seems to depend on the word category uniqueness point. Friederici et al. [13] demonstrated that, for morphologically complex words in which the word category was marked only on the suffix (e.g. refine versus refinement), the left anterior negativity started $50 \mathrm{~ms}$ after the mean word category uniqueness point (corresponding to $370 \mathrm{~ms}$ after the word onset). Interestingly, an early negative component with an anterior maximum has also been observed in correlation with the processing of closed class words [25,31-33,36,43].

In addition to the early negativities just described, many studies have observed anterior negativities with a left maximum and present approx. 300-500 ms after target onset (LAN). These effects have been observed quite systematically in correlation with morphosyntactic violations $[6,19,20]$. With respect to the temporal structure of language processing, the available evidence suggests that information about a word's class (open versus closed) and a word's category (e.g. noun versus verb) appears to be processed earlier than other types of syntactic information.

If a syntactic negativity was observed, it was often followed by a centro-parietal positivity generally referred to as the P600 component. The P600 was first found in correlation with the processing of syntactically non-preferred structures, i.e. during the processing of so-called 'garden path' sentences [38]. For these sentences it was observed as the only component. By contrast, for sentences containing outright violations $[6,13,19,20]$, it was often found to follow a syntactic negativity. With respect to its topography and timing, the P600 has some resemblance with the P300, a component manifesting a variable peak latency of between 300 and $800 \mathrm{~ms}$ which is elicited by a variety of non-linguistic cognitive tasks $[7,40]$. This similarity has given rise to some debate as to whether the P600 is a domain specific component or not $[6,19,22,37]$. Independently of the question of whether the P600 exclusively reflects linguistic processing aspects, its correlation with structural processing during comprehension is well established. Hence, it can be used as a valuable tool for examining such processes. In sum, selectional restriction (i.e. semantic) violations are usually correlated with an N400 component, while phrase structure violations are usually correlated with an early anterior negativity followed by a late positivity.

The present study makes use of these signatures in the ERP in order to examine the temporal structure of semantic integration processes and phrase structure processing during auditory sentence comprehension. In addition to a 'pure' semantic violation (2) and a 'pure' syntactic (phrase structure) violation condition (3), we also studied a violation condition in which the critical word induced both a phrase structure violation and a selectional restriction violation (4) (see Table 1).

The critical word, i.e. the word on which an error became overt, was always the sentence final participle. We used passive voice sentences in which, in the verb-final language German, the verb obligatorily appears in sentence final position in matrix clauses (1). In the semantic condition (2), the verb could not be integrated into the prior sentence context due to a violation of its selectional requirements. In the syntactic condition (3), the participle was immediately preceded by a preposition, thus inducing a phrase-structure violation given that the noun phrase required by the preposition was missing. In the combined 
Table 1

Experimental conditions and example sentences with approximate literal translations in parenthesis (The critical word is in italic)

Experimental sentences

(1) Correct

(2) Semantically incorrect

(3) Syntactically incorrect

(4) Semantically and syntactically incorrect
Das Brot wurde gegessen.

(The bread was eaten)

Der Vulkan wurde gegessen.

(The volcano was eaten)

Das Eis wurde im gegessen.

(The ice cream was in-the eaten)

Das Türschloß wurde im gegessen.

(The door lock was in-the eaten)

Filler sentences

(5) Correct

Die Pizza wurde im Restaurant gegessen (The pizza was in-the restaurant eaten)

condition (4), a semantic and a syntactic violation were realized simultaneously on the same target word. In addition to correct sentences without a prepositional phrase (1), we also included a filler condition containing sentences with a full prepositional phrase (5). This was done to prevent participants from anticipating the syntactic violation when processing the preposition.

The predictions for the pure semantic and the pure syntactic violation were straightforward. For the semantic condition, we expected to find an N400 component, while for the syntactic condition, we expected an early anterior negativity followed by a P600. Such results would replicate previous findings [13,14,21-23]. The prediction for the combined violation condition clearly depends on the assumptions made about the functional and temporal dependencies between syntactic and semantic processes. There are three possibilities, in principle. First, semantic and syntactic processes could be totally independent when occurring in temporal succession. If this was the case, we would expect a combination of all three ERP components: an early anterior negativity, an N400 and a P600. Second, if lexical-semantic information is used very rapidly and in close interaction with syntactic information during sentence processing, one would predict an early influence of the semantic properties of the word, i.e. the early negativity should differ between the combined violation condition and the pure syntactic violation condition $[28,44]$. Third, if the processes of phrase structure building are independent of semantic aspects but not vice versa $[9,10,17]$, one would predict (a) the early left anterior negativity to be unaffected by the semantic violation, whereas (b) the subsequent semantic integration processes reflected by the N400 should be affected by the phrase structure error as lexical integration is not licensed by the syntactic structure.

In our study, the sentences were presented as connected speech. Processing of auditory language has a preeminent status as compared to visual language processing, both phylo- and ontogenetically as well as in terms of the amount of input over life span [8]. For this reason, studying spoken language should prove particularly fruitful with respect to gaining an understanding of the basic principles underlying language comprehension.
In the following, we present two ERP experiments systematically varying syntactic and semantic well-formedness and differing only with respect to the instructions given to the participants. While participants judged the sentences for their overall correctness in experiment 1 , they judged the same sentences for semantic coherence in experiment 2. This change of attentional focus towards semantic aspects makes it possible to examine the relative amount of automaticity of the processes reflected by the different ERP components. An ERP component remaining unaffected by this change of instruction can be viewed as a reflection of more automatic and non-strategic processes than a component which is affected.

\section{Experiment 1}

\subsection{Participants}

Sixteen right-handed students of the Free University of Berlin (12 female, age range 19-35 years, mean 25 years) participated in the study. One participant had to be replaced because of a large number of DC drift artifacts. All participants were native speakers of German and had no known hearing deficit. They were either paid or received course credits for participation.

\subsection{Materials}

Participial forms of 100 different transitive verbs served as target words in all experimental conditions. All began with the regular German participial morpheme 'ge-'. For each participle, five different sentences were constructed according to the schema presented in Table 1, thus resulting in 500 experimental sentences. Two hundred of these sentences ( 40 from each condition) were presented to each participant. Each of the participles occurred in two different conditions; these were systematically varied and counterbalanced over subsets of items and subgroups of participants, such that each participle contributed to each experimental condition equally often and each participant received items from all experimental conditions equally 
often. The correct condition including a full prepositional phrase served as a filler condition as it was the only condition in which the correctness of the sentence could already be anticipated before encountering the participle. Therefore, the correct condition without a prepositional phrase (hereafter: correct condition) was used to evaluate the specific effects for the three incorrect conditions.

Additional sentences were included for the following reasons. On the one hand, we aimed at having an approximately equal number of correct and incorrect sentences within a session. On the other hand, we wanted to ensure that the probability of the word following the auxiliary being a participle (correct and semantic condition) would be equal to that of it being a preposition (syntactic and combined condition). To this purpose, we selected 20 additional participles, ten of which were used for creating sentences of type (1) and (2), while the other ten were used for creating sentences of type (1) and (5). These 40 additional sentences were the same in all experimental lists. They were not averaged in the ERP to avoid differences in the signal-to-noise ratio across conditions. This procedure resulted in an overall ratio of $46 \%$ correct sentences to $54 \%$ incorrect sentences. Of all these, $46 \%$ contained an auxiliary-participle transition and 54\% contained an auxiliary-preposition transition. Correspondingly, each participant received the following number of sentences per condition: 60 in the correct condition, 50 in the semantic condition, 40 in the syntactic condition, 40 in the combined condition, and 50 in the correct condition including a prepositional phrase.

The sentences were presented in a pseudo-randomized order which was obtained using the following constraints. First, sentences from the same condition were not presented in more than three consecutive trials. Second, no more than four correct or incorrect sentences were presented in succession. Third, at least 30 trials intervened between repetitions of the same participle. We created five experimental lists which were pseudo-randomized independently and presented forwards as well as backwards to compensate for possible effects of target repetition. All ten randomizations were presented at least once but not more than twice across the sixteen participants.

All sentences were spoken by a female native speaker of German. The sentences were recorded on digital-audio tape and then sampled at $20 \mathrm{kHz}$ with a 16-bit resolution. In order to ensure for a precise time locking of the ERP in each individual sentence, the onset of each word was marked by way of a careful inspection of the auditory and visual signal. Finally, it is possible that having to pronounce a syntactically malformed sentence may lead to such a sentence manifesting acoustic or prosodic anomalies. In order to prevent such effects occurring prior to the participle in the syntactically incorrect sentences, the speaker produced these sentences with a noun following the preposition. This noun always had the same onsetphoneme as the participle and ended in a phoneme which was identical to the last phoneme of the preposition occurring in that sentence. The sentences to be used in the syntactic violation conditions were then created by eliminating the noun from the sentences produced as described above, thus avoiding an unnatural acoustic transition due to coarticulation differences [22].

\subsection{Acceptability rating of the stimulus material}

Thirty-five participants (who did not participate in the ERP experiments) performed a semantic acceptability rating of all experimental sentences, which were presented to them in different pseudo-randomized sequences. The aim of this rating was to guarantee for a comparable semantic acceptability across the different conditions, i.e. to ensure that the semantic and the combined condition would be judged as equally unacceptable and that the correct and the syntactic condition would be judged as equally acceptable in semantic terms. Because participants were instructed to judge the sentences for semantic acceptability only, we presented only syntactically correct sentences during the acceptability rating, i.e. we omitted the preposition in the syntactic and the combined condition. The sentences were presented in written form and the task of the participant was to indicate on a five-point scale how well the sentence final word would fit in the prior sentence context $(1=$ not acceptable; $5=$ highly acceptable). These ratings revealed that the semantic acceptability varied across different conditions (mean ratings: correct: 4.03 (S.D. $=0.17$ ); syntactic: 3.97 (S.D. $=0.17$ ); semantic: 1.03 (S.D. $=0.17$ ); combined: 1.03 (S.D. $=0.17$ )). In line with our predictions, the judgments for the correct and the syntactic condition did not differ significantly $(t(34)=1.44, \quad P=0.16)$, nor did the judgments for the semantic and the combined condition.

\subsection{Procedure}

Participants were seated in a comfortable chair approximately $100 \mathrm{~cm}$ in front of a computer screen. The structure of each trial was as follows. A fixation point appeared on the CRT $500 \mathrm{~ms}$ before the auditory sentence presentation started and remained on the screen until $3000 \mathrm{~ms}$ after the end of the auditory presentation. The spoken sentence was presented binaurally via headphones. Then, a response signal was presented on the screen for $2000 \mathrm{~ms}$. The next trial started after an inter-trial interval of $1000 \mathrm{~ms}$. Participants were asked to avoid blinks and other movements during the presentation of the fixation point. They held a response box with both hands and were asked to judge the sentences for overall correctness by pressing one of two buttons with their thumbs during the presentation of the response signal. The delayed judgment ensured that the ERP to the critical word was not affected by motor responses. The instructions did not differentiate between the different types of errors. Before the session began, an 
example sentence was given for each of the five sentence types. Sentences were presented in five blocks each containing 48 trials. Prior to the experimental blocks, 15 practice sentences were presented. The whole experiment lasted approximately $2 \mathrm{~h}$.

\subsection{ERP recording}

The EEG was recorded with 19 tin electrodes secured in an elastic cap (Electro Cap International) and placed in the following locations: Fz, Cz, Pz, F7/8, F3/4, FT7/8, FC3/ 4, CP5/6, P3/4, P7/8, O1/2 [42]. The vertical electrooculogram (VEOG) was recorded from electrodes placed above and below the right eye. The horizontal EOG (HEOG) was recorded from positions at the outer canthus of each eye. The recordings were referenced against the left mastoid. The activity over the right mastoid was actively recorded and did not reveal any condition specific variation. Off-line, all recordings were re-referenced to the average of the two mastoid recordings. The AFZ electrode served as ground. Electrode impedance was kept below 3 $\mathrm{k} \Omega$. The biosignals were amplified within a bandpass from DC to $40 \mathrm{~Hz}$ and digitized with $250 \mathrm{~Hz}$.

\subsection{Data analysis}

Only trials with correct responses were analyzed. All trials were evaluated individually for EOG or other artifacts and trials contaminated by artifacts were excluded from the averaging procedure. On average, less than ten trials in each condition had to be excluded; these were equally distributed across conditions (correct: 7.4 (S.D.= 4.7); semantic condition: 6.3 (S.D.=5.6); syntactic condition: 8.9 (S.D. $=5.0$ ); combined condition: 9.8 (S.D. $=$ 6.9)). Event-related potentials were computed separately for each participant and each experimental condition for $1500 \mathrm{~ms}$ after the onset of the participle relative to two different baselines. Due to the fact that our experimental conditions differed with respect to the word immediately preceding the critical word, determining a prestimulus baseline would have been problematic. Therefore, we chose to use a 100-ms post-stimulus onset baseline $[13,22,34]$. The description of our results focuses on this main analysis. To allow for an evaluation of the results using the more common prestimulus onset baseline, we additionally computed averages relative to a $100-\mathrm{ms}$ prestimulus baseline. The results of the statistical analyses listed in the tables are provided for each of the two baselines. To anticipate the outcome, the choice of baseline has only a minor impact on the overall pattern of results.

For the statistical analysis of the behavioral data, error rates were computed separately for each condition. For a statistical evaluation of the ERP effects, the average voltage amplitudes in pre-defined time windows relative to the onset of the participle were computed for the three violation conditions and the correct condition. The follow- ing time windows were defined: $100-250 \mathrm{~ms}$ for the early negativity; 400-700 ms for the N400 component and $300-1000 \mathrm{~ms}$ for the late positivity. The selection of these time windows was based on the latency of the components in question in previous studies using auditory presentation and on a visual inspection of the grand averages.

All analyses were computed using the multivariate approach to repeated measurement $[35,46]$ and followed a hierarchical analysis schema. Each violation condition was evaluated separately against the correct condition. To allow for a quantification of hemispheric differences, the three midline positions and the lateral positions were analyzed separately. For the midline electrodes the analysis included the variables condition (correct vs. incorrect) and electrode (Fz, Cz, Pz). Rather than the variable electrode, the analyses for the lateral electrodes included two topographical variables which were completely crossed: ant-pos (anterior vs. posterior) and hemisphere (left vs. right). For the two levels of the variable ant-pos, eight anterior electrode positions (F7, F3, FT7, FC3, F8, F4, FT8, FC4) and eight posterior positions (CP5, P3, P7, O1, CP6, P4, $\mathrm{P} 8, \mathrm{O} 2)$ were chosen, while the electrodes for the variable hemisphere were F7, F3, FT7, FC3, CP5, P3, P7, O1 (left) versus F8, F4, FT8, FC4, CP6, P4, P8, O2 (right). When the variable condition revealed an at least marginally significant interaction $(P<0.10)$ with one or both of the topographical variables, a further analysis was conducted on a lower level, i.e. for each quadrant separately, with the variables condition and electrode.

In addition, we carried out separate analyses of variance for each electrode position and condition for 30 consecutive time-intervals of $50 \mathrm{~ms}$ length each to ensure that no possible effects may be overlooked [19,20]. To minimize type 1 errors, significance of an effect will be assumed whenever two or more successive interval-analyses reached a significance level of $<0.05$. These results are incorporated in Fig. 1.

\section{Results}

\subsection{Behavioral data}

Error rates were low (correct: $1.8 \%$; semantic: $4.8 \%$; syntactic: $2.0 \%$; combined: $1.6 \%$ ). Although there was a marginally significant condition effect $(F(3,13)=2.58, P=$ 0.10 ), Neuman-Keuls pairwise comparisons did not reveal any significant differences between conditions.

\subsection{ERP data}

The ERP data for the critical participle are displayed in Fig. 1. Correct sentences elicited a negative potential peaking around $400 \mathrm{~ms}$ after the onset of the participle at centro-parietal electrode sites followed by a small parietal positivity. The negativity can be described as an N400 


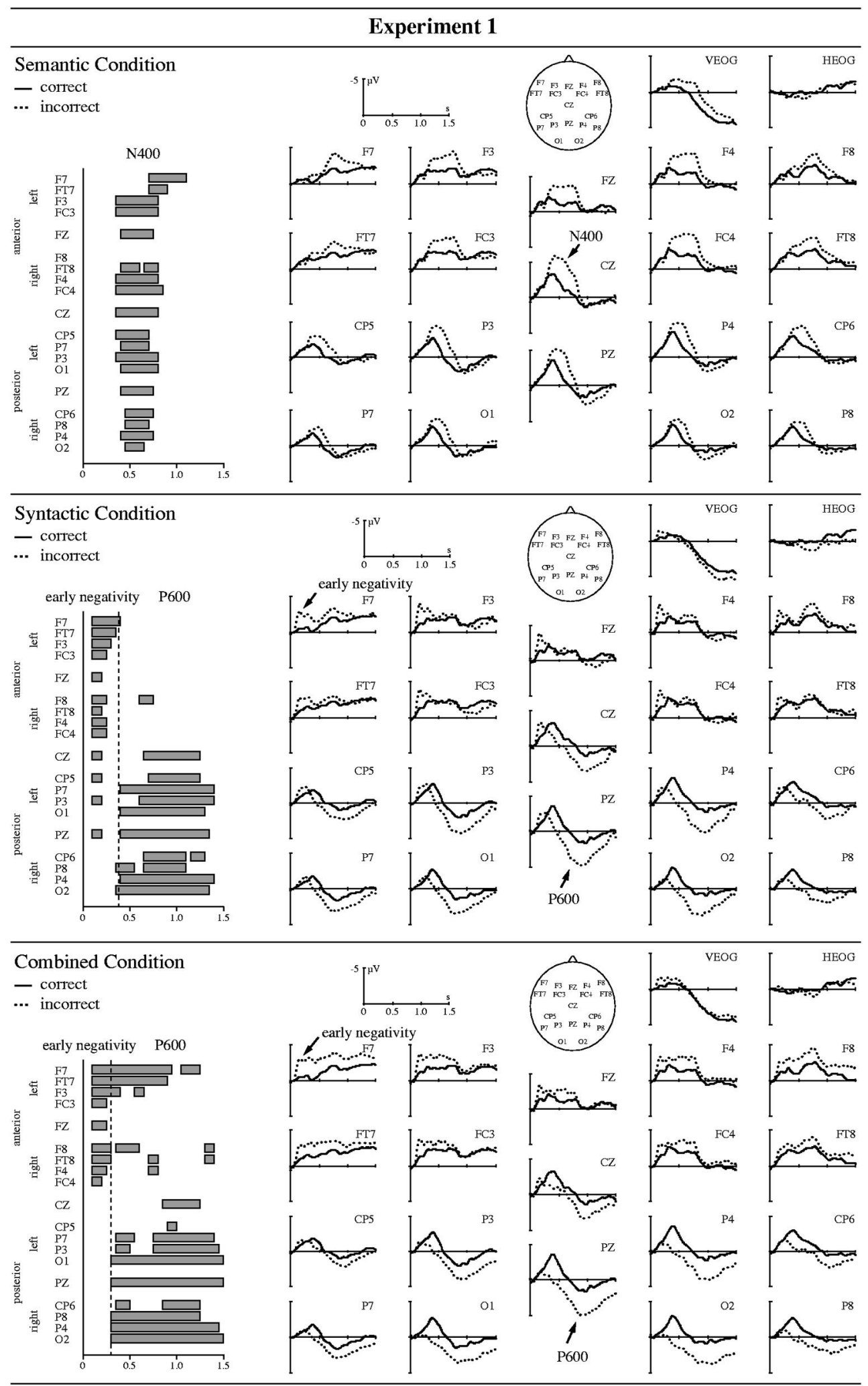

Fig. 1. Grand average ERPs for experiment 1. Participants judged the sentences for overall correctness. In this and the following figure, the averages are calculated relative to a $100-\mathrm{ms}$ post-stimulus onset baseline. The origin of the $x$-axis corresponds to the onset of the participle and negative voltage is plotted upwards. Upper row: ERPs for the semantic violation condition (e.g. 'Der Vulkan wurde gegessen') as compared to the correct condition (e.g. 'Das Brot wurde gegessen'). Middle row: ERPs for the syntactic phrase structure violation condition (e.g. 'Das Eis wurde im gegessen') as compared to the correct condition. Lower row: ERPs for the combined semantic and syntactic violation condition (e.g. 'Das Türschloß wurde im gegessen') as compared to the correct condition. The left part of each row displays the results of MANOVAs comparing the incorrect condition to the correct condition for each electrode, starting at the onset of the participle. Shaded bars indicate significant effects $(P<0.05)$. Effects are only marked whenever two or more successive 50-ms windows revealed a reliable effect. 
Table 2

Global analyses of ERP data for each experimental condition in experiment 1

\begin{tabular}{|c|c|c|c|c|c|c|c|c|c|c|c|}
\hline \multirow[t]{3}{*}{ Source } & \multirow[t]{3}{*}{ df } & \multirow{2}{*}{\multicolumn{2}{|c|}{$\frac{\text { Semantic }}{400-700}$}} & \multicolumn{4}{|l|}{ Syntactic } & \multicolumn{4}{|l|}{ Combined } \\
\hline & & & & \multicolumn{2}{|l|}{$100-250$} & \multicolumn{2}{|l|}{$300-1000$} & \multicolumn{2}{|l|}{$100-250$} & \multicolumn{2}{|l|}{$300-1000$} \\
\hline & & $F$ & M.S.E. & $F$ & M.S.E. & $F$ & M.S.E. & $F$ & M.S.E. & $F$ & M.S.E. \\
\hline Co (post-stimulus) & 1,15 & $21.10 * * *$ & 19.98 & $23.60 * * *$ & 6.80 & 2.07 & 30.94 & $8.28 * *$ & 8.47 & $<1$ & - \\
\hline Co (pre-stimulus) & 1,15 & $16.02 * * *$ & 29.61 & $5.86^{* *}$ & 10.57 & $4.14 *$ & 39.37 & $3.27 *$ & 16.98 & $<1$ & - \\
\hline $\mathrm{Co} \times \mathrm{He}$ (post-stimulus) & 1,15 & $<1$ & - & 1.62 & 1.58 & 1.30 & 2.07 & $6.12 * *$ & 1.26 & $<1$ & - \\
\hline $\mathrm{Co} \times \mathrm{He}$ (pre-stimulus) & 1,15 & 2.26 & 5.97 & 1.50 & 3.75 & 1.47 & 4.11 & 1.57 & 3.14 & $<1$ & - \\
\hline Co $\times$ Ap (post-stimulus) & 1,15 & $<1$ & - & $9.17 * * *$ & 4.44 & $21.75 * * *$ & 8.18 & $12.64 * * *$ & 7.63 & $28.16^{* * *}$ & 11.67 \\
\hline $\mathrm{Co} \times \mathrm{Ap}$ (pre-stimulus) & 1,15 & 1.67 & 8.00 & $11.70^{* * *}$ & 2.65 & $38.69 * * *$ & 4.05 & $10.64 * * *$ & 6.70 & $29.79 * * *$ & 9.42 \\
\hline $\mathrm{Co} \times \mathrm{He} \times \mathrm{Ap}$ (post-stimulus) & 1,15 & $5.42 * *$ & 0.86 & $<1$ & - & 1.35 & 0.47 & 1.01 & 0.45 & 1.78 & 1.26 \\
\hline $\mathrm{Co} \times \mathrm{He} \times \mathrm{Ap}$ (pre-stimulus) & 1,15 & $25.07 * * *$ & 0.42 & $<1$ & - & 1.53 & 0.61 & $<1$ & - & $7.01 * *$ & 0.82 \\
\hline
\end{tabular}

Note: Effects based on a 100-ms post-stimulus onset baseline and a 100-ms pre-stimulus baseline are displayed. Co, condition; He, hemisphere; Ap, anterior-posterior dimension; $* P<0.10, * * P<0.05$, *** $P<0.01$.

component reflecting the semantic integration of the sentence final word into the prior sentence context. As expected, the semantic violation condition elicited a much more pronounced N400 component than the correct sentences. The syntactic violation condition elicited an early anterior negativity which consisted of two peaks (at about 165 and $300 \mathrm{~ms}$ ) and which even extended to some parietal electrode sites (CP5, P3). The two peaks could also be observed for the correct condition, though with a reduced amplitude. Over some right frontal sites, however, the second peak reached nearly the same amplitude as in the syntactic condition. The event-related response for the combined condition was remarkably similar to that for the syntactic condition. Most importantly, this condition did not elicit an N400 component. The descriptive observations were supported by the subsequent analyses. As only the results of the variable condition are of theoretical interest in this study, the description of results will focus on effects involving this variable.

\subsection{Semantic condition}

The analyses for the time window 400-700 ms revealed a significant main effect of condition and a reliable threeway interaction of condition, hemisphere and ant-pos (see Tables 2-4), reflecting the fact that the negativity for

Table 3

Analyses of ERP data for each quadrant in each experimental condition in experiment 1

\begin{tabular}{|c|c|c|c|c|c|c|c|c|c|c|c|}
\hline \multirow[t]{3}{*}{ Source } & \multirow[t]{3}{*}{ df } & \multirow{2}{*}{\multicolumn{2}{|c|}{$\frac{\text { Semantic }}{400-700}$}} & \multicolumn{4}{|l|}{ Syntactic } & \multicolumn{4}{|l|}{ Combined } \\
\hline & & & & \multicolumn{2}{|l|}{$100-250$} & \multicolumn{2}{|l|}{$300-1000$} & \multicolumn{2}{|l|}{$100-250$} & \multicolumn{2}{|l|}{$300-1000$} \\
\hline & & $F$ & M.S.E. & $F$ & M.S.E. & $F$ & M.S.E. & $F$ & M.S.E. & $F$ & M.S.E. \\
\hline \multicolumn{12}{|l|}{ Left anterior } \\
\hline Co (post-stimulus) & 1,15 & $6.05^{* *}$ & 9.93 & $28.99 * * *$ & 3.77 & $<1$ & - & 28.38 *** & 4.13 & $6.02 * *$ & 9.45 \\
\hline Co (pre-stimulus) & 1,15 & 2.96 & 10.6 & $10.54 * * *$ & 5.99 & $<1$ & - & $13.53^{* * *}$ & 5.92 & 2.53 & 12.70 \\
\hline Co $\times \mathrm{El}$ (post-stimulus) & 3,13 & $3.58 * *$ & - & 1.89 & - & 2.44 & - & 1.13 & - & $3.15^{*}$ & - \\
\hline $\mathrm{Co} \times \mathrm{El}$ (pre-stimulus) & 3,13 & $4.16^{* *}$ & - & 1.40 & - & $2.57^{*}$ & - & $<1$ & - & 2.17 & - \\
\hline \multicolumn{12}{|l|}{ Right anterior } \\
\hline Co (post-stimulus) & 1,15 & $8.90 * * *$ & 14.18 & $18.01^{* *}$ & 4.11 & $<1$ & - & $11.64 * * *$ & 4.67 & $4.49^{*}$ & 10.52 \\
\hline Co (pre-stimulus) & 1,15 & $8.22 * *$ & 19.06 & $6.27 * *$ & 4.81 & $<1$ & - & $5.02 * *$ & 9.62 & $3.12 *$ & 13.33 \\
\hline $\mathrm{Co} \times \mathrm{El}$ (post-stimulus) & 3,13 & $4.95 * *$ & - & 1.52 & - & 1.12 & - & 1.63 & - & 1.42 & - \\
\hline Co $\times \mathrm{El}$ (pre-stimulus) & 3,13 & $3.16^{*}$ & - & $<1$ & - & $<1$ & - & $<1$ & - & $<1$ & - \\
\hline \multicolumn{12}{|l|}{ Left posterior } \\
\hline Co (post-stimulus) & 1,15 & $29.59 * * *$ & 4.38 & $4.85^{* *}$ & 3.01 & $8.65^{* *}$ & 10.31 & $<1$ & - & $6.89 * *$ & 11.99 \\
\hline Co (pre-stimulus) & 1,15 & $23.35^{* * * *}$ & 6.69 & 1.38 & 3.83 & $9.40 * * *$ & 12.79 & $<1$ & - & $5.74 * *$ & 13.14 \\
\hline Co $\times \mathrm{El}$ (post-stimulus) & 3,13 & $5.68 * *$ & - & 2.39 & - & 1.59 & - & 1.98 & - & $5.01^{* *}$ & - \\
\hline Co $\times \mathrm{El}$ (pre-stimulus) & 3,13 & $10.66 * * *$ & - & 2.07 & - & 2.23 & - & 1.69 & - & $5.32 * *$ & - \\
\hline \multicolumn{12}{|l|}{ Right posterior } \\
\hline Co (post-stimulus) & 1,15 & $22.89 * * *$ & 5.00 & 2.61 & 2.33 & $13.67 * * *$ & 10.33 & $<1$ & - & $11.95^{* * *}$ & 13.61 \\
\hline Co (pre-stimulus) & 1,15 & $21.97 * * *$ & 7.61 & $<1$ & - & $15.82 * * *$ & 13.01 & $<1$ & - & $13.38 * * *$ & 12.05 \\
\hline Co $\times \mathrm{El}$ (post-stimulus) & 3,13 & $3.73^{* *}$ & - & $2.97 *$ & - & $5.67 * *$ & - & 1.45 & - & $5.39 * *$ & - \\
\hline Co×El (pre-stimulus) & 3,13 & $3.69 * *$ & - & $<1$ & - & $3.66^{* *}$ & - & $<1$ & - & $4.05^{* *}$ & - \\
\hline
\end{tabular}

Note: Effects based on a 100 -ms post-stimulus onset baseline and a 100-ms pre-stimulus baseline are displayed. Co, condition; El, electrode; $* P<0.10$, $* * P<0.05, * * * P<0.01$. 
Table 4

Analyses of ERP data for the midline electrodes in each experimental condition in experiment 1

\begin{tabular}{|c|c|c|c|c|c|c|c|c|c|c|c|}
\hline \multirow[t]{3}{*}{ Source } & \multirow[t]{3}{*}{$\mathrm{df}$} & \multirow{2}{*}{\multicolumn{2}{|c|}{$\frac{\text { Semantic }}{400-700}$}} & \multicolumn{4}{|l|}{ Syntactic } & \multicolumn{4}{|c|}{ Combined } \\
\hline & & & & \multicolumn{2}{|l|}{$100-250$} & \multicolumn{2}{|l|}{$300-1000$} & \multicolumn{2}{|l|}{$100-250$} & \multicolumn{2}{|l|}{$300-1000$} \\
\hline & & $F$ & M.S.E. & $F$ & M.S.E. & $F$ & M.S.E. & $F$ & M.S.E. & $F$ & M.S.E. \\
\hline Co (post-stimulus) & 1,15 & $21.95 * * *$ & 8.98 & $10.83^{* * *}$ & 4.21 & $4.29^{*}$ & 13.93 & 2.03 & 4.53 & $3.29 *$ & 12.05 \\
\hline Co (pre-stimulus) & 1,15 & $17.64 * * *$ & 13.04 & 1.63 & 9.01 & $4.80^{* *}$ & 23.63 & $<1$ & - & $<1$ & - \\
\hline Co $\times E l$ (post-stimulus) & 2,14 & $5.10^{* *}$ & - & $<1$ & - & $7.36^{* * * *}$ & - & $4.21 * *$ & - & $10.68 * * *$ & - \\
\hline Co $\times E l$ (pre-stimulus) & 2,14 & 2.33 & - & $<1$ & - & $16.71 * * *$ & - & 2.61 & - & 2.65 & - \\
\hline
\end{tabular}

Note: Effects based on a 100-ms post-stimulus onset baseline and a 100-ms pre-stimulus baseline are displayed. Co, condition; El, electrode; $* P<0.10$, $* * P<0.05$, *** $P<0.01$.

incorrect as opposed to correct sentences was widely distributed but most pronounced over left posterior sites.

\subsection{Syntactic condition}

The analyses for the early time window (100-250 ms) revealed a main effect of condition and a significant interaction of condition and ant-pos (see Table 2). The analyses for each quadrant showed that the condition effect had its maximum over anterior scalp sites. The analyses for the second time window (300-1000 ms) showed a highly significant interaction of the variable condition with the variable ant-pos (see Tables 2-4), reflecting the posterior distribution of the positivity. In summary, the results for the syntactic condition demonstrated a highly reliable early negativity for anterior electrode locations. This effect was followed by a posterior positivity which was symmetrically distributed over the posterior scalp and which can be classified as a P600 component.

\subsection{Combined condition}

For the early time window (100-250 ms), the analyses showed a significant main effect of condition as well as an interaction of condition and hemisphere. Furthermore, there was a highly significant interaction of the variables condition and ant-pos, reflecting the anterior distribution of the negativity in this time window (see Tables 2-4). The posterior positivity observed within the second time window (300-1000 ms) was reflected in a significant interaction of condition and ant-pos (see Tables 2-4). In sum, the results for the combined condition revealed nearly the same data pattern as for the syntactic condition, i.e. an early anterior negativity and a late posterior positivity. No N400 was found in this condition.

\subsection{Comparison of the syntactic and the combined condition}

As the combined condition clearly differed from the semantic condition but resembled the syntactic condition, additional analyses were computed to directly compare the combined condition to the syntactic condition. A global analysis including the variables violation type (syntactic versus combined), hemisphere and ant-pos for the early time window (100-250 ms) revealed a marginal interaction of violation type and ant-pos $(F(1,15)=3.13, P<$ 0.10, M.S.E. =3.79). The analyses for each quadrant showed no significant differences for the two anterior quadrants $(F \mathrm{~s}<1)$, but a marginal main effect of violation type for the left posterior quadrant $(F(1,15)=3.84, P<$ 0.07 , M.S.E. $=3.18)$ and a reliable effect for the right posterior quadrant $(F(1,15)=5.08, P<0.05$, M.S.E. $=3.55)$, in which the syntactic condition was more negative than the combined condition.

With regard to the second time window (300-1000 ms), the analyses revealed a marginal interaction of violation type and ant-pos $(F(1,15)=3.87, P<0.07$, M.S.E. =5.94). However, the analyses for the four quadrants did not show reliable effects of violation type (left anterior: $F(1,15)=$ 2.68, $P<0.13$, M.S.E. $=7.40$; right anterior: $F(1,15)=2.73$, $P<0.12$, M.S.E. $=7.84$; both posterior quadrants: $F<1$ ).

In summary, the direct comparison between the syntactic and the combined condition showed that these two conditions elicited remarkably similar ERP patterns. The only exceptions to this were a sustained negativity at F7 and FT7 and differences at posterior electrode sites in the early time window, in which the syntactic condition was slightly more negative than the combined condition.

\section{Discussion}

The ERPs for the simple violation conditions replicated previous results. Phrase structure violations elicited an early anterior negativity peaking at about $165 \mathrm{~ms}$ which was followed by a P600 component. The early anterior negativity did not lateralize to the left. A lateralization was found in some of the earlier work using similar stimulus materials [13,14], but not in other studies [15,21,23]. To what extent such distributional variations reflect individual or functional differences has to be explored by future research. Concerns might be raised as to whether the early anterior negativity is caused by prosodic properties rather than by syntactic features. There are a number of counterarguments to this objection. First of all, a similar early 
anterior negativity was observed in an experiment in which the same stimuli were presented visually [18]. Second, two previous experiments using naturally produced syntactically incorrect sentences gave rise to a similar early anterior negativity $[13,14]$. Third, detailed analyses of duration and fundamental frequency (F0) of the complete sentence material showed that these were largely comparable. Fourth, a behavioral discrimination test demonstrated that for the overwhelming majority of spliced syntactically incorrect sentences participants were not able to detect the splice and classified them as sounding 'natural'.

Semantic violations elicited an N400 component. Importantly, despite of the semantic anomaly, no N400 was elicited when the syntactic and the semantic violations were combined within the same sentence. This pattern suggests that sentences containing a phrase structure violation were processed on a syntactic level only: the semantic information influenced neither the early negativity nor the P600. The observation that the combined violation condition did not elicit an N400 replicates findings from a recent study in which sentences were presented visually [15]. Assuming that the N400 component reflects semantic integration processes, the present finding suggests that, in the absence of phrase structure integrity, the critical lexical element was not semantically integrated into the prior sentence context. This interpretation is not only supported by the fact that there was no N400 difference between the correct and the combined condition, but also by a comparison of the correct and the syntactic condition. The correct condition elicited an N400 which is taken to reflect the lexical semantic integration of the participle into the short, low constraining sentence context. A rather unrestrictive semantic context such as the present one (as also revealed by the semantic acceptability judgment experiment; see Section 2.3.), leads to a rather pronounced N400 activity. Interestingly, however, no such N400 activity was seen for the syntactically incorrect sentences, suggesting that the verb was not integrated into the sentence context on a semantic level. When considered together, these data suggest that the early syntactic processes are not influenced by the lexical-semantic properties of the word. However, these early syntactic processes seem to be able to block semantic integration processes of the current word whenever lexical integration is not licensed by the phrase structure.

The finding that the early negativity was not influenced by the lexical-semantic information might appear obvious when considering the temporal structure of the different ERP components and the availability of the different information types. Semantic processes are usually thought to be reflected by the N400 component, which is present only after the early negativity. Therefore, one need not necessarily expect the early negativity to be influenced by the $\mathrm{N} 400$, as the early syntactic process may already have been completed by the time the semantic process starts. However, there are at least two counterarguments to such a view. First, as we used connected speech, lexical-semantic information might have been available fairly early due to coarticulation information speeding up word recognition. For example, Holcomb and Neville [24] showed that the existence of such 'nonsemantic between-word contextual cues' (p. 297) can lead to N400 effects present as early as $200 \mathrm{~ms}$ allowing, in principle, for an early influence of lexical-semantic information on syntactic word category processing. Second, there might be semantic processes invisible to the ERP which occur prior to those reflected in the N400, a component thought to reflect processes of semantic integration, i.e. late aspects of lexical processing. Behavioral evidence shows that, in addition to these late semantic integration processes, there are also earlier lexical processes which are behaviorally connected with automatic semantic priming effects [30]. There is as yet no known electrophysiological marker of automatic semantic priming, and thereby of the early aspects of lexical processing. Still, these early semantic processes could, in principle, influence early syntactic processes. These considerations render our finding far from trivial.

From a methodological point of view, the result for the combined condition might raise concerns about a possible temporal overlap of the $\mathrm{N} 400$ and the late positivity. However, as the late positivity did not differ between the pure syntactic and the combined condition, such an objection is not tenable. A component overlap could only be a possibility if the syntactic condition had also elicited an N400 component, yet the wealth of evidence on parsing in the ERP literature renders this highly unlikely. Moreover, visual inspection of single subject averages revealed that this is not the case. Another possible concern with respect to the dissociation of an N400 effect in the semantic and in the combined condition is that these conditions might differ in the degree of semantic violation. However, the results of the behavioral semantic acceptability rating do not support such a view. Furthermore, one might speculate that the insertion of the preposition in the combined condition should increase the semantic violation perceived, thus leading to a higher degree of semantic violation in the combined condition than in the pure semantic condition. However, if this were true, our claim that the missing N400 component is not caused by a component overlap of $\mathrm{N} 400$ and P600 would be strengthened even further, as we would then expect to find a bigger N400 component in the combined than in the pure semantic condition.

The results presented so far indicate that the semantic integration of a word into the prior sentence context is not initiated automatically, but rather depends on the wellformedness of the syntactic phrase structure. This raises questions regarding the possible strategic nature of semantic processes. Studies examining word-word priming effects have shown that a modulation of the N400 component is dependent on attentional mechanisms [2,4,5]. This could also hold for semantic integration in a senten- 
tial context, i.e. participants may not engage in semantic integration once a phrase structure violation has been detected. Note that the task used in experiment 1 did not specify which linguistic information should lead participants to classify sentences of the combined condition as being incorrect. Participants could, in principle, have given a correct response solely on the basis of either information, i.e. phrase structure information or semantic information. As the ERP response to phrase structure violations in the syntactic condition (early negativity) was elicited earlier than the response to semantic violations in the semantic condition (N400), participants might have been able to classify a sentence in the combined condition as incorrect solely on the basis of the syntactic information (which is available earlier).

If temporal aspects are primarily responsible for the observed pattern of results and the combined condition does not elicit an N400 because the system generally acts on the basis of syntactic information without getting engaged in semantic integration processes, this pattern should be independent of task demands. If, however, attentional aspects are primarily responsible, then the combined condition should give rise to an N400 if participants are confronted with instructions and a task necessitating the processing of the semantic aspects of the sentence. Thus, a task requiring a semantic analysis of the sentence-final words for accurate performance should lead to a modulation of the N400 component in the combined condition.

This possibility was examined in experiment 2 . In this experiment, we explicitly instructed our participants to ignore syntactic violations and focus on the semantic coherence of the sentences only. This means that each sentence required the verb's argument(s) and its selectional restrictions to be checked against one another and the verb to be semantically integrated into the prior context. Such processes can easily be conducted for the correct and semantic conditions. In the syntactic condition, the verb can also easily be integrated into the sentence context when the violation is ignored because the sentence context is in agreement with the selectional restrictions of the verb. As the task explicitly requires the syntactic violation to be ignored, participants must internally correct the sentence before matching the verb with the first noun phrase on the basis of the verb's selectional restrictions. Theoretically, two strategies are possible: either the preposition could be deleted or the missing noun could be inserted. Under the attend-to-semantic task, we predict an N400 component for the combined condition of comparable size to that elicited by the semantic condition. For these two conditions, the sentence context does not fulfill the selectional restrictions of the verb, thus causing difficulties in lexical integration which, in turn, should lead to a pronounced N400 component.

The task variation in experiment 2 also enables us to examine properties of the early syntactic processes with respect to one additional detail. In Hahne and Friederici [22] it was proposed that the early negativity reflects a relatively automatic process. If this is valid, the early negativity should not be influenced by the varying attentional demands imposed by the instructions. Therefore, we expected the early negativity to be present again for both the syntactic condition and the combined condition, and to be of equal size as in experiment 1 .

\section{Experiment 2}

\subsection{Participants}

Sixteen right-handed students of the University of Leipzig were recruited from the subject pool of the Max Planck Institute (11 female, age range: 19-28 years, mean: 23 years) and were paid for participation. All were native speakers of German and had no known hearing deficit.

\subsection{Procedure}

The procedure was identical to the one used in experiment 1 , except that the instructions differed. In the present experiment, participants were asked to judge the sentences only for their semantic coherence, i.e. to decide whether the sentence made sense or not, and to disregard structure violations. As in experiment 1, participants were given example sentences of each of the five different sentence types and the corresponding correct answers. Unlike in experiment 1, stimuli were presented via loudspeakers instead of over headphones. The average sound pressure level ranged from approximately 63 to $67 \mathrm{~dB}$.

\subsection{Materials}

The same stimuli and randomizations as in experiment 1 were used.

\subsection{ERP recording}

The electrophysiological recording was identical to that in experiment 1 .

\subsection{Data analysis}

The procedure for analyzing the data was the same as in experiment 1. Rejected trials were equally distributed across conditions (correct: 6.9 (S.D.=3.4); semantic condition: 6.9 (S.D.=3.8); syntactic condition: 6.9 (S.D. = 3.6); combined condition: 7.8 (S.D.=4.1)). Additionally, analyses comparing the two experiments were carried out. 


\section{Results}

\subsection{Behavioral data}

Error rates were low (correct: $0.7 \%$; semantic: $2.1 \%$; syntactic: $1.4 \%$; combined: $2.5 \%$ ) and did not differ reliably across conditions $(F(3,13)=1.50, P=0.26)$.

\subsection{ERP data}

The ERP data for the critical participle are displayed in Fig. 2. As in experiment 1, correct sentences elicited an N400 and a subsequent positivity. The positivity was, however, more pronounced than in the previous experiment. As in experiment 1 , semantic violations elicited a pronounced N400 component. In contrast to the previous experiment, however, no N400 difference between correct and incorrect sentences was observable over left anterior sites. The syntactic condition again elicited an early anterior negativity, but unlike in experiment 1, syntactic violations also elicited an N400-like negativity over centro-parietal sites which was comparable to that observable for the correct condition. This effect was followed by a late positivity which was only slightly more positive than that in the correct condition. The combined condition evoked responses qualitatively different to those in experiment 1 . In addition to an early anterior negativity, it now also elicited an N400 component relative to the correct condition. The N400 effect for the combined violation was of comparable size to that in the semantic condition.

\subsection{Semantic condition}

The analysis for the time window 400-700 ms revealed a significant main effect of condition and reliable interactions of this variable with hemisphere as well as with ant-pos (see Tables 5-7), reflecting the fact that the N400 component had a posterior maximum and was more pronounced over the right than over the left hemisphere.

\subsection{Syntactic condition}

Statistical analyses performed on the early time window (100-250 ms) revealed a reliable interaction of condition and ant-pos, reflecting the fact that the negativity was restricted to anterior electrode sites (see Tables 5-7). Within the late time window used in the analyses of experiment 1 (300-1000 ms), only the interaction of condition and ant-pos was marginally reliable (see Table 5). However, subsequent analyses per quadrant revealed that the condition effect was not reliable in any of the quadrants (see Table 6), nor were any condition effects for the midline positions (see Table 7). As the small positivity was visible in the data from about 500 to $1200 \mathrm{~ms}$, we additionally evaluated this time window. This analysis showed that the interaction of condition and ant-pos approached significance $(F(1,15)=4.38, P<0.06$, M.S.E. $=$ 2.37). Subsequent analyses revealed that the condition effect was significant for the left posterior quadrant $(F(1,15)=4.62, P<0.05$, M.S.E. $=2.04)$ but not for the right posterior quadrant $(F(1,15)=2.68, P<0.12$, M.S.E. $=$ 2.32). There were no significant effects for the midline electrodes. In sum, a highly reliable bilateral early negativity could be observed, while there was only a tendencial difference between correct and incorrect sentences with regard to the late positivity.

\subsection{Combined condition}

Similar to the syntactic condition, the analyses of the early time window (100-250 ms) for the combined condition showed a significant interaction of condition and ant-pos (see Tables 5 and 6). The analyses of the midline positions did not reveal any significant effects in this time window (see Table 7). The N400 component found in this condition was analyzed in the time window 400-700 ms. The analysis of the lateral electrodes revealed only a reliable main effect of condition but no significant interactions (see Table 5). Therefore, no further analyses were conducted for these electrodes. The midline electrodes also showed a main effect of condition. In summary, the combined condition elicited an anterior negativity followed by a widely distributed N400 component.

\subsection{Comparison of the syntactic and the combined condition (100-250 ms)}

As the syntactic condition and the combined condition elicited similar early anterior negativities, a direct comparison was conducted for the early time window, as for experiment 1 . These analyses did not show any reliable differences between the two conditions.

\subsection{Comparison of the semantic and the combined condition (400-700 ms)}

A direct comparison of the N400 component in the semantic and the combined condition revealed a significant interaction of violation type and ant-pos $(F(1,15)=12.04$, $P<0.01$, M.S.E. $=0.78)$. Analyses for each quadrant showed that the difference between these two conditions was mainly restricted to the right posterior quadrant $(F(1,15)=7.88, \quad P<0.05$, M.S.E. $=0.19)$, with semantic violations yielding a more negative ERP than combined violations.

\subsection{Analyses across the two experiments}

A direct comparison of the ERPs for the two experiments was performed with experiment as between-subject variable and condition (correct, semantic violation, syntac- 


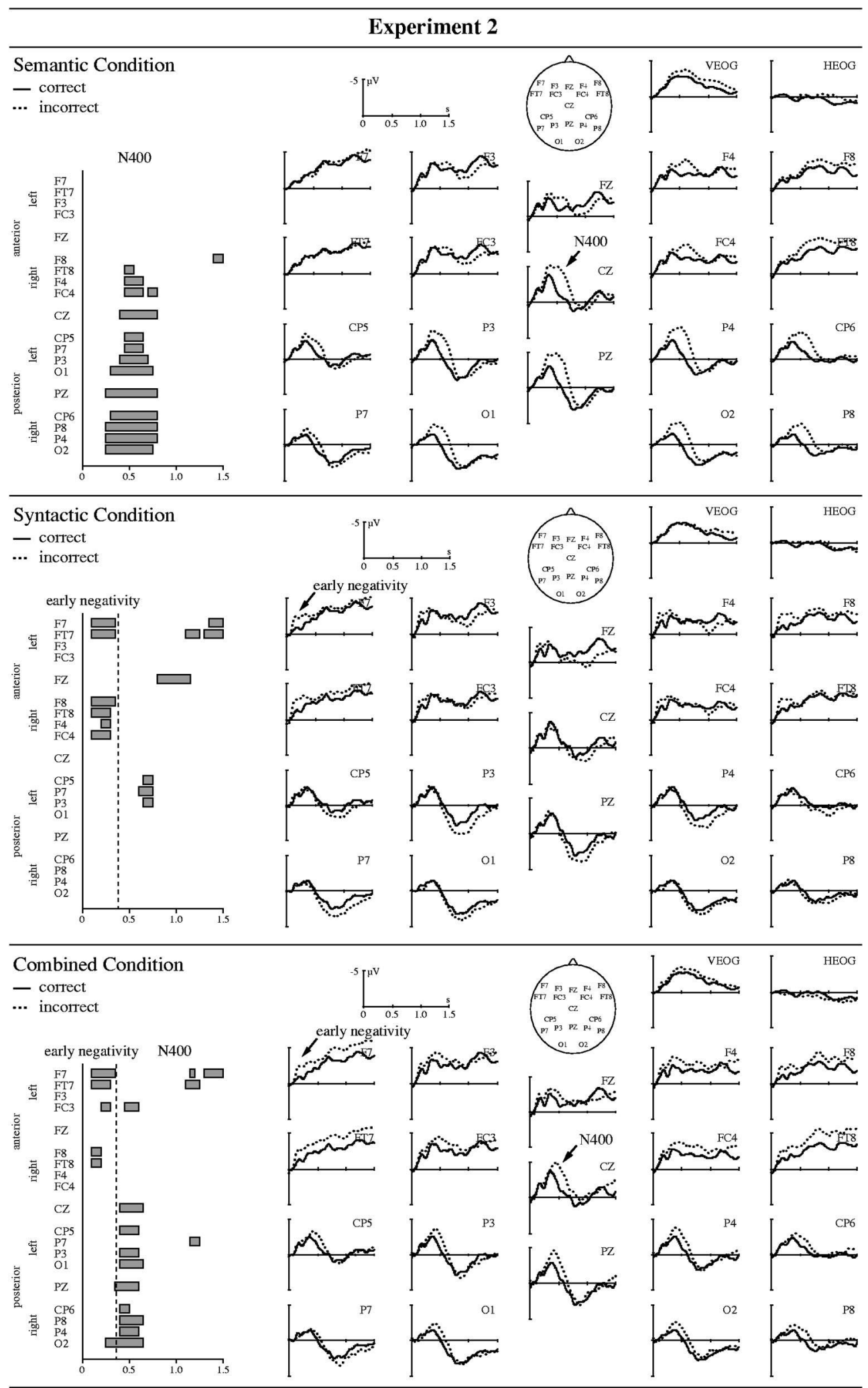

Fig. 2. Grand average ERPs for experiment 2. Participants judged the sentences for semantic coherence. For further details see legend of Fig. 1.

tic violation, combined violation) as a within-subject variable. These analyses were restricted to those electrode sites and time windows that had revealed significant condition effects in the analyses for the individual experiments.

The analysis of the early negativity $(100-250 \mathrm{~ms})$ for 
Table 5

Global analyses of ERP data for each experimental condition in experiment 2

\begin{tabular}{|c|c|c|c|c|c|c|c|c|c|c|c|}
\hline \multirow[t]{3}{*}{ Source } & \multirow[t]{3}{*}{ df } & \multirow{2}{*}{\multicolumn{2}{|c|}{$\frac{\text { Semantic }}{400-700}$}} & \multicolumn{4}{|l|}{ Syntactic } & \multicolumn{4}{|l|}{ Combined } \\
\hline & & & & \multicolumn{2}{|l|}{$100-250$} & \multicolumn{2}{|c|}{$300-1000$} & \multicolumn{2}{|l|}{$100-250$} & \multicolumn{2}{|l|}{$400-700$} \\
\hline & & $F$ & M.S.E. & $F$ & M.S.E. & $F$ & M.S.E. & $F$ & M.S.E. & $F$ & M.S.E. \\
\hline Co (post-stimulus) & 1,15 & $23.29^{* * * *}$ & 10.85 & 2.95 & 8.63 & $<1$ & - & $7.66^{* *}$ & 6.18 & $6.93 * *$ & 23.76 \\
\hline Co (pre-stimulus) & 1,15 & $18.30^{* * * *}$ & 19.48 & 2.45 & 12.90 & $<1$ & - & $<1$ & - & $6.02 * *$ & 22.12 \\
\hline $\mathrm{Co} \times \mathrm{He}$ (post-stimulus) & 1,15 & $12.66^{* * * *}$ & 1.77 & $<1$ & - & $<1$ & - & $<1$ & - & $<1$ & - \\
\hline $\mathrm{Co} \times \mathrm{He}$ (pre-stimulus) & 1,15 & $3.60^{*}$ & 3.00 & 2.35 & 2.10 & 1.40 & 1.79 & $3.86^{*}$ & 2.03 & 1.22 & 3.77 \\
\hline Co $\times$ Ap (post-stimulus) & 1,15 & $7.99^{* *}$ & 8.77 & $7.94 * *$ & 3.95 & $3.31 *$ & 7.11 & $11.96^{* * *}$ & 5.20 & $<1$ & - \\
\hline Co $\times$ Ap (pre-stimulus) & 1,15 & $10.74 * * *$ & 10.95 & $7.09 * *$ & 8.30 & $3.41^{*}$ & 6.21 & $5.30^{* *}$ & 10.73 & $<1$ & - \\
\hline $\mathrm{Co} \times \mathrm{He} \times \mathrm{Ap}$ (post-stimulus) & 1,15 & $<1$ & - & $<1$ & - & $<1$ & - & $<1$ & - & 2.08 & 0.56 \\
\hline Co $\times$ He $\times$ Ap (pre-stimulus) & 1,15 & $<1$ & - & $<1$ & - & 2.41 & 0.75 & 2.47 & 0.56 & $6.98^{* *}$ & 0.45 \\
\hline
\end{tabular}

Note: Effects based on a 100-ms post-stimulus onset baseline and a 100-ms pre-stimulus baseline are displayed. Co, condition; He, hemisphere; Ap, anterior-posterior dimension; $* P<0.10, * * P<0.05$, $* * * P<0.01$.

Table 6

Analyses of ERP data for each quadrant in each experimental condition in experiment 2

\begin{tabular}{|c|c|c|c|c|c|c|c|c|c|}
\hline \multirow[t]{3}{*}{ Source } & \multirow[t]{3}{*}{$\mathrm{df}$} & \multirow{2}{*}{\multicolumn{2}{|c|}{$\frac{\text { Semantic }}{400-700}$}} & \multicolumn{4}{|l|}{ Syntactic } & \multirow{2}{*}{\multicolumn{2}{|c|}{$\frac{\text { Combined }}{100-250}$}} \\
\hline & & & & \multicolumn{2}{|l|}{$100-250$} & \multicolumn{2}{|l|}{$300-1000$} & & \\
\hline & & $F$ & M.S.E. & $F$ & M.S.E. & $F$ & M.S.E. & $F$ & M.S.E. \\
\hline \multicolumn{10}{|l|}{ Left anterior } \\
\hline Co (post-stimulus) & 1,15 & $<1$ & - & $9.24 * * *$ & 5.73 & $<1$ & - & $4.31 * *$ & 7.52 \\
\hline Co (pre-stimulus) & 1,15 & $<1$ & - & $7.23^{* *}$ & 9.16 & $<1$ & - & $5.41 * *$ & 10.94 \\
\hline $\mathrm{Co} \times \mathrm{El}$ (post-stimulus) & 3,13 & $3.56^{* *}$ & _- & 1.81 & - & $3.95^{* *}$ & - & $2.99 *$ & - \\
\hline $\mathrm{Co} \times \mathrm{El}$ (pre-stimulus) & 3,13 & $4.64 * *$ & - & $3.35^{*}$ & - & $8.75^{* * *}$ & - & $3.43^{* *}$ & - \\
\hline \multicolumn{10}{|l|}{ Right anterior } \\
\hline Co (post-stimulus) & 1,15 & $6.27 * *$ & 6.73 & $19.13^{* * * *}$ & 2.93 & $<1$ & - & $7.99 * *$ & 3.06 \\
\hline Co (pre-stimulus) & 1,15 & $4.84 * *$ & 7.22 & $5.60^{* * *}$ & 4.75 & $<1$ & - & 1.83 & 7.59 \\
\hline Co $\times \mathrm{El}$ (post-stimulus) & 3,13 & $2.97^{*}$ & - & $3.96^{* *}$ & - & 2.15 & - & 1.84 & - \\
\hline $\mathrm{Co} \times \mathrm{El}$ (pre-stimulus) & 3,13 & $3.14 *$ & - & 1.66 & - & 1.21 & - & 1.43 & - \\
\hline \multicolumn{10}{|l|}{ Left posterior } \\
\hline Co (post-stimulus) & 1,15 & $21.09 * * *$ & 4.87 & $<1$ & - & 2.05 & 7.84 & $<1$ & - \\
\hline Co (pre-stimulus) & 1,15 & $16.15^{* * *}$ & 11.24 & $<1$ & - & 1.64 & 9.46 & $<1$ & - \\
\hline $\mathrm{Co} \times \mathrm{El}$ (post-stimulus) & 3,13 & $9.66 * * *$ & - & $4.11^{* *}$ & - & $2.73^{*}$ & - & $3.57^{* *}$ & - \\
\hline $\mathrm{Co} \times \mathrm{El}$ (pre-stimulus) & 3,13 & $6.51^{* * *}$ & - & 1.69 & - & 1.60 & - & 2.56 & - \\
\hline \multicolumn{10}{|l|}{ Right posterior } \\
\hline Co (post-stimulus) & 1,15 & $47.48^{* * *}$ & 4.21 & $<1$ & - & 1.45 & 6.29 & $<1$ & - \\
\hline Co (pre-stimulus) & 1,15 & $27.27 * * *$ & 9.69 & $<1$ & - & 1.80 & 9.72 & 1.14 & 6.13 \\
\hline Co $\times \mathrm{El}$ (post-stimulus) & 3,13 & $4.89 * *$ & - & 2.39 & - & $<1$ & - & $<1$ & - \\
\hline $\mathrm{Co} \times \mathrm{El}$ (pre-stimulus) & 3,13 & $3.83^{* *}$ & - & $<1$ & - & $<1$ & - & $<1$ & - \\
\hline
\end{tabular}

Note: Effects based on a 100-ms post-stimulus onset baseline and a 100-ms pre-stimulus baseline are displayed. Co, condition; El, electrode; $* P<0.10$, $* * P<0.05$, *** $P<0.01$.

Table 7

Analyses of ERP data for the midline electrodes in each experimental condition in experiment 2

\begin{tabular}{|c|c|c|c|c|c|c|c|c|c|c|c|}
\hline \multirow[t]{3}{*}{ Source } & \multirow[t]{3}{*}{ df } & \multirow{2}{*}{\multicolumn{2}{|c|}{$\frac{\text { Semantic }}{400-700}$}} & \multicolumn{4}{|c|}{ Syntactic } & \multicolumn{4}{|c|}{ Combined } \\
\hline & & & & \multicolumn{2}{|c|}{$100-250$} & \multicolumn{2}{|c|}{$300-1000$} & \multicolumn{2}{|c|}{$100-250$} & \multicolumn{2}{|c|}{$400-700$} \\
\hline & & $F$ & M.S.E. & $F$ & M.S.E. & $F$ & M.S.E. & $F$ & M.S.E. & $F$ & M.S.E. \\
\hline Co (post-stimulus) & 1,15 & $32.68 * * *$ & 4.49 & $<1$ & - & 1.83 & 5.95 & 1.13 & 2.46 & $7.84 * *$ & 8.23 \\
\hline Co (pre-stimulus) & 1,15 & $21.81 * * *$ & 8.68 & $<1$ & - & 1.57 & 11.92 & $<1$ & - & $3.94^{*}$ & 10.91 \\
\hline $\mathrm{Co} \times \mathrm{El}$ (post-stimulus) & 2,14 & $6.65 * * *$ & - & 1.25 & - & $<1$ & - & 2.33 & - & $5.63 * *$ & - \\
\hline $\mathrm{Co} \times \mathrm{El}$ (pre-stimulus) & 2,14 & $4.19 * *$ & - & $3.18^{*}$ & - & $<1$ & 1.01 & - & - & $4.26^{* *}$ & - \\
\hline
\end{tabular}

Note: Effects based on a 100-ms post-stimulus onset baseline and a 100-ms pre-stimulus baseline are displayed. Co, condition; El, electrode; $* P<0.10$, $* * P<0.05$, *** $P<0.01$. 
the anterior lateral electrode positions revealed a significant main effect of condition $(F(3,28)=16.08, P<0.001)$, but neither experiment nor the interaction of the two variables were reliable $(F \mathbf{s}<1)$. In summary, there were no differences between experiments within the early time window.

The comparison of the two experiments in the N400 time window (400-700 ms) was conducted for the midline electrodes (as the $\mathrm{N} 400$ was most pronounced at $\mathrm{Cz}$ ). It revealed a significant main effect of condition $(F(3,28)=$ $17.69, P<0.01)$ and a reliable interaction of condition and experiment $(F(3,28)=3.22, P<0.05)$, but the main effect of experiment was not significant $(F<1)$. At the single electrode level, only the combined condition showed a reliable difference between the two experiments at $\mathrm{Cz}$ (correct: $t(30)=0.48, P=0.64$; semantic: $t(30)=0.95, P=$ 0.35 ; syntactic: $t(30)=0.70, P=0.49$; combined: $t(30)=$ 2.20, $P=0.04$ ).

The direct comparison between experiments in the P600 window (300-1000 ms) was also conducted for the midline positions. It showed a reliable effect of condition $(F(3,28)=9.63, \quad P<0.01)$, but no significant effect of experiment $(F<1)$. Furthermore, it revealed a reliable interaction of condition $\times$ experiment $(F(3,28)=3.44, P<$ $0.05)$ as well as a significant interaction of condition $X$ experiment $\times$ electrode $(F(6,25)=5.24, P<0.01)$, reflecting the fact that the condition effects differed between the two experiments at parietal rather than frontal electrode positions. For the electrode site $\mathrm{Pz}$, a highly significant effect was obtained for the combined condition $(t(30)=2.50$, $P=0.02)$. The other conditions did not differ across the two experiments (correct: $t(30)=1.27, P=0.21$; semantic: $t(30)=0.68, P=0.50$; syntactic: $t(30)=0.85, P=0.40)$. In summary, in the time window used to evaluate the late positivity in experiment 1 (overlapping with the N400 time window), it is again the combined condition which clearly differs between experiments.

\section{Discussion}

The aim of experiment 2 was to evaluate the impact of task demands on sentence comprehension strategies. While in experiment 1 neither syntax nor semantics was specifically emphasized, the instructions given in experiment 2 required participants to focus on semantics while ignoring structural violations. The main results of this experiment can be summarized as follows: The task-induced emphasis on semantics did not affect the early anterior negativity, neither in the syntactic nor in the combined condition. The task did not affect the N400 for the semantic condition either. However, it had a clear influence on both the N400 and the P600 in the syntactic and the combined condition. While in experiment 1 , sentences with a combined violation were associated with a late positivity, in experiment 2 they elicited only an N400 of similar magnitude to the N400 in the semantic violation condition. Furthermore, in experiment 2, sentences containing only a phrase structure violation did not differ from correct sentences with respect to the late positivity, but elicited an N400 activity similar to that observed for the correct sentences.

The obtained pattern of results is fully compatible with our hypotheses. The data suggest that semantic integration processes are not initiated automatically in the case of a phrase structure violation, but can be initiated by attentional mechanisms. Such semantic integration processes were observed for both types of syntactically incorrect sentences in experiment 2. The N400-like activity in the syntactic condition suggests that participants were able to integrate the word into the prior sentence on a lexical-semantic level in a similar way as in the correct sentences when instructed to ignore the syntactic violation. The observed N400 activity might be taken as evidence that participants internally deleted the preposition, thus yielding a representation comparable to that for correct sentences without a prepositional phrase rather than inserting a noun to make the prepositional phrase complete. Combined violations elicited an N400 activity which was of about the same size as that for the pure semantic violation condition. This effect was highly reliable. The finding of a pronounced N400 in the combined condition in experiment 2 rules out the caveats mentioned with regard to the interpretation of the data from experiment 1 . The present experiment clearly demonstrates (a) that the experimental stimuli are indeed able to elicit an N400 and (b) that the absence of such a component in experiment 1 is not likely to be due to a component overlap of N400 and P600.

The results of experiment 2 suggest that the processes underlying the early syntactic negativity possess a high degree of autonomy. The early anterior negativity was observed in both the pure syntactic condition and the combined condition. This replication demonstrates once more that the lexical-semantic information of a word does not influence this very early syntactic process. Rather, the early negativity elicited by phrase structure errors persisted even when participants focused their attention on semantic aspects of the sentence.

While the results concerning the early negativity and the N400 appear to be particularly clear, things are more complicated with respect to the late positivity. Despite its presence in the syntactic condition in experiment 1 , this component could not be reliably observed in the syntactic condition in experiment 2 in which the attentional focus was on semantic aspects. This renders an interpretation of the P600 effect in the syntactic condition somewhat difficult. The direct ERP comparison of correct and syntactically incorrect sentences in experiment 2 did not reveal a significant difference between these conditions, possibly indicating that the P600 for incorrect sentences is reduced when there is an attentional focus on semantics. However, a conjoined analysis of experiments 1 and 2 
revealed that neither the syntactic violation condition nor the correct condition differed significantly across experiments, thus suggesting that the shift of attentional focus via instruction did not influence the brain response to syntactically incorrect sentences.

In summary, the task manipulation employed in experiment 2 clearly influenced the later components. It did not, however, affect the early anterior negativity.

\section{General discussion}

The aim of the present study was to examine how syntactic and semantic analysis processes in auditory language comprehension are temporally coordinated and what the functional dependencies between these processes are. In particular, we used event-related brain potentials to investigate whether and how phrase structure information and semantic information influence each other. We presented sentences which violated either semantic aspects of the sentence, syntactic aspects, or both, and compared the ERPs elicited by the critical verb participle in these different types of incorrect sentences to those appearing in correct sentences. In previous research, phrase structure violations have been shown to elicit an early anterior negativity, presumably reflecting first-pass sentence parsing processes. One critical question was whether this early anterior negativity would be influenced by lexical-semantic information. While interactive models of language comprehension would predict an early influence of semantic information on this process [28,44], serial models would predict no such influence $[9,10,17]$. A second question was whether an early detection of a syntactic violation can block on-line lexical-semantic integration processes, and if so whether this effect could be overcome by directing the attentional focus onto semantic processing. The results were clear-cut.

\subsection{Effects of semantic information and semantic instruction}

In both ERP experiments, phrase structure violations elicited an early anterior negativity which was independent of whether there was an additional semantic violation. Experiment 2 further demonstrated that this syntactic negativity persisted even when participants focused their attention on semantic information. The ERP data indicate that neither semantic information as such nor attentional processes induced by the semantic coherence judgment modulate the early negativity and thus the very early syntactic processing stage it reflects. Finally, the data clearly suggests that words inducing a phrase structure violation are not semantically integrated into the sentence unless participants are instructed to do so.

The observation that sentences in the combined violation condition did not elicit an N400 component unless the instruction required participants to evaluate the sentences at a semantic level has important implications. It shows that the processing of phrase structure information has priority over that of lexical-semantic information and that the syntactic feature of an incorrect word category may block the semantic integration of that particular word. Most interestingly, this blocking effect can be overcome by an experimentally induced attentional focus on semantics, thus suggesting that the semantic integration of a word into its sentential context can be characterized as a process which is subject to strategic or controlled processing.

This result is in agreement with data from a recent study by Friederici et al. [15], who investigated a combined semantic-syntactic violation during reading. The syntactic violation was also a word category violation. As in the present study, the authors found no N400 for a combined violation suggesting a primacy of the processing of phrase structure information. This data pattern is extended by a recent study from Frisch et al. [16]. Here, it is suggested that, in the case of a phrase structure violation, further processing is blocked not only with regard to the semantic aspects of a verb, but also with regard to its argument structure [12].

Interestingly, an early negativity followed by a P600 was also observed for phrase structure violations in sentences consisting of pseudowords, but in which the morphological markers were retained to signal the word's category [23]. Thus, the comprehension system does not seem to attempt at integrating the element eliciting a phrase structure violation on a semantic level, regardless of whether the element is a word that can easily be integrated into the prior sentence context or a pseudoword for which no semantic integration is possible.

In sum, the present data show that the different ERP components under examination differ remarkably with respect to their susceptibility to experimental variations. The early anterior negativity reflects an early syntactic process which is independent of semantic information processing, task demands, probability manipulations and the lexical status of the element [22,23], suggesting a high degree of automaticity for the processing of phrase structure information.

By contrast, the late components, N400 and P600, were clearly dependent on task demands and instructions. The finding that the $\mathrm{N} 400$ depends on task parameters indicates that the process underlying this component in sentence comprehension should be characterized as a rather controlled process. Although this has already been claimed for the N400 in single word processing [2,4,5,41], it had not yet been shown in sentential contexts.

\subsection{Combined semantic-syntactic violations across different studies}

At a first glance, the present findings combining seman- 
tic and phrase structure violations seem to be partly in conflict with other recent ERP data from reading experiments combining semantic and morphosyntactic violations. Gunter et al. [19] found an N400 and a left anterior negativity but a reduced P600 for the combined semanticmorphosyntactic condition as compared to the morphosyntactic violation. More recently, Gunter et al. [20] conducted a reading experiment crossing a gender violation with semantic predictability (cloze probability) of noun targets. In this study, the gender error elicited a LAN component and an N400 for the combined condition. These two components did not affect each other. A subsequent P600 was, however, influenced by the syntactic and the semantic variable.

The conflicting findings between these studies and the present one may be reconciled if one considers the types of syntactic violations examined (phrase structure violations versus morphosyntactic violations) and the timing of the associated ERP components observed. Language-related ERP effects are observed in three different time windows. The first time window (approximately 100-300 ms) is characterized by very early syntactic negativities, the second time window (approximately 300-500 ms) comprises semantic N400 components as well as syntactic negativities (often left anterior: 'LAN effects'), and the third time window (approximately around $600 \mathrm{~ms}$ ) can be characterized by syntactic positivities ('P600 effects').

While the present study observed an early negativity, the effects in other studies using combined violations became manifest only in the second and third time windows. Gunter and co-workers $[19,20]$ observed a LAN and an N400 in the same time range and found an effect on the subsequent P600. Taking these studies together, it seems that the cognitive processes underlying an ERP component in the first time window are able to influence (and even block) the processing in the second time window (present study). In a similar way, the processes occurring in the second time window seem to be able to influence the processing in the third time window $[19,20]$. Thus, it seems that different processes within the same time window (N400 and LAN) are conducted independently, whereas processes performed in an earlier phase are able to influence processes taking place in a subsequent phase, but not vice versa. Hence, the very early negativity may block the N400, and N400/LAN components may influence the P600.

How do the findings from Ainsworth-Darnell et al. [1] and Osterhout and Nicol [39] fit into this view? These authors found independent effects for the N400 and the P600 for their combined semantic-syntactic conditions. While the critical element in the Ainsworth-Darnell et al. [1] study was a combination of a semantic violation and a syntactically non-preferred (but correct) reading, the critical element in the Osterhout and Nicol [39] study was a combination of a semantic violation and a syntactically incongruent suffix. Why was there no influence of the second phase (N400) on the third phase (P600) as seen by Gunter and co-workers $[19,20]$ ? It seems that a reduction of the P600 is only observed for combined semantic and syntactic anomalies if both semantic and syntactic aspects have been visibly processed during the second processing phase. In both studies in question, however, there was no reliable syntactic ERP component within the second time window. Therefore, one might speculate that, in those cases where combined violations do not elicit a syntactic negativity within the second processing phase, the P600 seems to be unaffected. This implies that, although the LAN and the N400 appear to be independent from one another during the second phase, the system evaluates these two parallel information channels conjointly before entering the third phase (P600).

\subsection{Implications for language comprehension models}

What conclusions can be drawn for models of language comprehension on the basis of these data? The findings of the present study are compatible with structure-driven serial models which assume an autonomous first-pass parse based on word category information $[9,10,17]$. Apart from the observation that the semantic information did not influence the early processing stage (as we did not find any difference between the syntactic and the combined violation in the early time window), the data also suggest that, in the case of a phrase structure error, semantic processes have no influence on the processing of that particular word at any time. The processing of a word's categorial information and its integration into the developing phrase structure of the sentence seems to occur extremely early during the processing of an individual word and to have a preeminent status in sentence comprehension.

This early availability of word category information differs from a situation in which syntactic information comes into play at about the same time as lexical-semantic information. This could be due to the fact that the critical information is available only at the end of a word, e.g. with the suffix whereas lexical-semantic information is available with the word stem or only accessible once all the relevant information encoded in a lexical entry is activated (e.g. grammatical gender). In such a case, semantic and syntactic processes seem to be 'carried out in parallel and independently' $[19,20]$. These parallel processes may influence late syntactic processes-as shown in experiments by Gunter and co-workers where combined semantic-syntactic anomalies elicited a LAN and an N400 but a reduced P600. Finally, if syntactic information comes into play only at a late stage, i.e. after semantic integration has taken place [1,39], the two informational sources may be processed independently (see also Ref. [23] for a discussion of this issue).

In conclusion, the combined data provide support for the notion that psycholinguistic modeling has to move away from focusing on what has been labeled the 'Great Divide', 
i.e. autonomy versus interaction [3]. The proposal advanced here is to seek for a more fine-grained analysis of the different subprocesses involved, both with regard to their temporal and with regard to their functional characteristics. The electrophysiological approach seems to provide for a possible integrative approach to behavioral data supporting syntax-first modular theories on the one hand and those supporting interactive models on the other hand. A possible integrative view would consider syntax-first modular aspects of processing to be connected to the early time window and interactive aspects of processing to come into play only during a late processing phase.

\section{Acknowledgements}

We are indebted to Shlomo Bentin, Ina Bornkessel, Jörg Jescheniak, Amy Schafer, Shari Speer, and an anonymous reviewer for their constructive comments and suggestions on earlier versions of this manuscript, and to Stefan Frisch and Heike Böthel for their help in data collection.

\section{References}

[1] K. Ainsworth-Darnell, H.G. Shulman, J.E. Boland, Dissociating brain responses to syntactic and semantic anomalies: evidence from event-related potentials, J. Mem. Lang. 38 (1998) 112-130.

[2] S. Bentin, M. Kutas, S. Hillyard, Semantic processing and memory for attended and unattended words in dichotic listening: behavioral and electrophysiological evidence, J. Exp. Psychol. 21 (1995) 5467.

[3] J.E. Boland, A. Cutler, Interaction with autonomy: multiple output models and the inadequacy of the great divide, Cognition 58 (1996) 309-320.

[4] C. Brown, P. Hagoort, The processing nature of the N400: evidence from masked priming, J. Cogn. Neurosci. 5 (1993) 34-44.

[5] D.J. Chwilla, C. Brown, P. Hagoort, The N400 as a function of the level of processing, Psychophysiology 32 (1995) 274-285.

[6] S. Coulson, J.W. King, M. Kutas, Expect the unexpected: eventrelated brain responses to morphosyntactic violations, Lang. Cogn. Processes 13 (1998) 21-58.

[7] M. Fabiani, G. Gratton, D. Karis, E. Donchin, The definition, identification, and reliability of measurement of the P300 component of the event-related brain potential, in: P.K. Ackles, J.R. Jennings, M.G.H. Coles (Eds.), Advances in Psychophysiology, Vol. 1, JAI Press, Greenwich, CT, 1987.

[8] F. Ferreira, M. Anes, Why study spoken language, in: M.A. Gernsbacher (Ed.), Handbook of Psycholinguistics, Academic Press, San Diego, 1994, pp. 33-56.

[9] L. Frazier, Sentence processing: a tutorial review, in: M. Coltheart (Ed.), Attention and Performance, Vol. XII, Erlbaum, Hillsdale, NJ, 1987, pp. 559-586.

[10] A.D. Friederici, The time course of syntactic activation during language processing: a model based on neuropsychological and neurophysiological data, Brain Lang. 50 (1995) 259-281.

[11] A.D. Friederici, Towards a neural basis of auditory sentence processing, Trends Cogn. Sci. (in press).

[12] A.D. Friederici, S. Frisch, Verb argument structure processing: the role of verb-specific and argument-specific information, J. Mem. Lang. 43 (2000) 476-507.

[13] A.D. Friederici, A. Hahne, A. Mecklinger, Temporal structure of syntactic parsing: early and late event-related brain potential effects elicited by syntactic anomalies, J. Exp. Psychol. 22 (1996) 12191248.

[14] A.D. Friederici, E. Pfeifer, A. Hahne, Event-related brain potentials during natural speech processing: effects of semantic, morphological and syntactic violations, Cogn. Brain Res. 1 (1993) 183-192.

[15] A.D. Friederici, K. Steinhauer, S. Frisch, Lexical integration: sequential effects of syntactic and semantic information, Mem. Cogn. 27 (1999) 438-453.

[16] S. Frisch, A. Hahne, A.D. Friederici, Argument-structure information defeated: ERP evidence for the autonomy of phrase-structure in sentence processing, in: Poster presented at the Conference on Architectures and Mechanisms for Language Processing (AMLaP), Edinburgh, Scotland, 1999, September.

[17] P. Gorrell, Syntax and Parsing, Cambridge University Press, Cambridge, UK, 1995.

[18] T.C. Gunter, A.D. Friederici, A. Hahne, Brain responses during sentence reading: visual input affects central processes, NeuroReport 10 (1999) 3175-3178.

[19] T.C. Gunter, L.A. Stowe, G. Mulder, When syntax meets semantics, Psychophysiology 34 (1997) 660-676.

[20] T.C. Gunter, A.D. Friederici, H. Schriefers, Syntactic gender and semantic expectancy: ERPs reveal early autonomy and late interaction, J. Cogn. Neurosci. 12 (2000) 556-568.

[21] A. Hahne, What's different in second language processing? Evidence from event-related brain potentials, J. Psychol. Res. 30 (2001) 251-266.

[22] A. Hahne, A.D. Friederici, Electrophysiological evidence for two steps in syntactic analysis: early automatic and late controlled processes, J. Cogn. Neurosci. 11 (2) (1999) 193-204.

[23] A. Hahne, J.D. Jescheniak, What's left if the Jabberwock gets the semantics? An ERP investigation into semantic and syntactic processes during auditory sentence comprehension, Cogn. Brain Res. 11 (2001) 199-212.

[24] P.J. Holcomb, H.J. Neville, Natural speech processing: an analysis using event-related brain potentials, Psychobiology 19 (1991) 286300.

[25] J.W. King, M. Kutas, Neural plasticity in the dynamics of human visual word recognition, Neurosci. Lett. 244 (1998) 1-4.

[26] M. Kutas, S.A. Hillyard, Reading senseless sentences: brain potentials reflect semantic incongruity, Science 207 (1980) 203-205.

[27] M. Kutas, C. Van Petten, Psycholinguistics electrified. Event-related brain potential investigations, in: M.A. Gernsbacher (Ed.), Handbook of Psycholinguistics, Academic Press, San Diego, 1994, pp. 83-143.

[28] M.C. MacDonald, N.J. Pearlmutter, M.S. Seidenberg, The lexical nature of syntactic ambiguity resolution, Psychol. Rev. 101 (1994) 676-703.

[29] D.C. Mitchell, Sentence parsing, in: M.A. Gernsbacher (Ed.), Handbook of Psycholinguistics, Academic Press, San Diego, 1994, pp. 375-409.

[30] J.H. Neely, Semantic priming effects in visual word recognition: a selective review of current findings and theories, in: D. Besner, G. Humphreys (Eds.), Basic Processes in Reading: Visual Word Recognition, Erlbaum, Hillsdale, NJ, 1991, pp. 264-336.

[31] H.J. Neville, D. Bavelier, Specificity and plasticity in neurocognitive development, in: M.S. Gazzaniga (Ed.), The New Cognitive Neurosciences, MIT Press, Cambridge, MA, 2000, pp. 83-98.

[32] H.J. Neville, S.A. Coffey, D.S. Lawson, A. Fischer, K. Emmorey, U. Bellugi, Neural systems mediating American Sign Language: effects of sensory experience and age of acquisition, Brain Lang. 57 (1997) 285-308.

[33] H.J. Neville, D.L. Mills, D.S. Lawson, Fractionating language: different neural subsystems with different sensitive periods, Cereb. Cortex 2 (1992) 244-258.

[34] H.J. Neville, J. Nicol, A. Barss, K.I. Forster, M.F. Garrett, Syntactically based sentence processing classes: evidence from event-related brain potentials, J. Cogn. Neurosci. 3 (1991) 151-165. 
[35] R.G. O'Brian, M.K. Kaiser, MANOVA method for analyzing repeated measures designs: an extensive primer, Psychol. Bull. 97 (1885) 316-333.

[36] L. Osterhout, On the brain response to syntactic anomalies: manipulations of word position and word class reveal individual differences, Brain Lang. 59 (1997) 494-522.

[37] L. Osterhout, P. Hagoort, A superficial resemblance does not necessarily mean you are part of the family: counterarguments to Coulson, King, and Kutas (1998) in the P600/SPS-P300 debate, Lang. Cogn. Processes 14 (1999) 1-14.

[38] L. Osterhout, P.J. Holcomb, Event-related potentials and syntactic anomaly: evidence of anomaly detection during the perception of continuous speech, Lang. Cogn. Processes 8 (1993) 413-437.

[39] L. Osterhout, J. Nicol, On the distinctiveness, independence, and time course of the brain responses to syntactic and semantic anomalies, Lang. Cogn. Processes 14 (1999) 283-317.

[40] W.S. Pritchard, Psychophysiology of P300, Psychol. Bull. 89 (1981) 506-540.

[41] M.D. Rugg, Event-related brain potentials dissociate repetition effects of high- and low- frequency words, Mem. Cogn. 18 (1990) 367-379.

[42] F. Sharbrough, G.E. Chatrian, R.P. Lesser, H. Lüders, M. Nuwer, T.W. Picton, American Electroencephalographic Society Guidelines for standard electrode position nomenclature, J. Clin. Neurophysiol. 8 (1991) 200-202.

[43] M. Te Keurs, C.M. Brown, P. Hagoort, D.F. Stegeman, Electrophysiological manifestation of open- and closed-class words in patients with Broca's aphasia with agrammatic comprehension, Brain 122 (1999) 839-854.

[44] J.C. Trueswell, M.K. Tanenhaus, S.M. Garnsey, Semantic influences on parsing: use of thematic role information in syntactic disambiguation, J. Mem. Lang. 33 (1994) 285-318.

[45] C. Van Petten, M. Kutas, Influences of semantic and syntactic context on open and closed class words, Mem. Cogn. 19 (1991) 95-112.

[46] M.W. Vasey, J.F. Thayer, The continuing problem of false positives in repeated measures ANOVA in psychophysiology: a multivariate solution, Psychophysiology 24 (1997) 479-486. 